\title{
Tachyonic Quintessential Inflation
}

\author{
Víctor H. Cárdenas* \\ Departamento de Física y Matemática, Universidad Andres Bello, Los Fresnos 52, Vina del Mar, Chile
}

\begin{abstract}
We study the possibility to construct an observationally viable scenario where both early Inflation and the recently detected accelerated expansion of the universe can be explained by using a single scalar field associated with the Tachyon. The Reheating phase becomes crucial to enable us to have a consistent cosmology and also to get a second accelerated expansion period. A discussion using an exponential potential is presented.
\end{abstract}

PACS numbers: $98.80 . \mathrm{Cq}$

\section{INTRODUCTION}

The ultimate goal for a cosmological model is to make all the observational data available consistent within its framework. In this context, a model which try to describe the current accelerated expansion must also be consistent with large scale structure observations, cosmic background measurements and the standard model of cosmology. In this paper I propose a model to describe both the current cosmic accelerated expansion and also the early phase known as inflation 1] consistent with the standard model of cosmology.

Models of this type have been proposed in the past and are usually called "Quintessential Inflationary" models 2]. They are characterized by a single scalar field which evolve in a non oscillatory potential. The field drives both accelerated phases by rolling down the potential to an asymptotic flat region. The contact with the standard model of cosmology is made by introducing a reheating phase - where almost all the particles in the universe were created - through a gravitational particle production process [3]. The shape of the potential has to be adjusted to ensure a kinetic dominated phase during which the scalar field contribution becomes negligible and the structure formation and nucleosynthesis processes works without any interference. The advantage of using a single scalar field to describe both accelerated phases is lost when we introduce an ad-hoc potential, adjusting its shape. Actually, this procedure leads to a problem of consistency with the amplitude of density perturbations called the $\eta$ problem [4].

In this paper I propose a similar model which describe both periods of cosmic acceleration by a single scalar field which we identify with the tachyon leading to what we called "Tachyonic Quintessential Inflation" model. As far as I know, the tachyon has been used to study cosmology in different contexts; studying inflation, dark energy and also as a candidate to model dark matter and dark energy together. Here I present a distinct model; a single framework to describe inflation and dark energy. The main advantage of using the tachyon as the field driven

\footnotetext{
${ }^{*}$ Electronic address: vcardenas[at]unab.cl
}

both phases is not only the economy of scalar fields used, but also that we know from first principles the shape of the scalar potential to be used [5, 6].

Tachyons and inflation have been studied in several papers [7, 8, 9]. The idea is very simple but some of them are fine tuned and have a problem of consistency with the scale of energy density fluctuations 8]. We refer to section II for a discussion about that. Tachyons and dark energy have been considered a couple of times [10]. We can understand that because the usual exit from tachyonic inflation leads to an asymptotic equation of state $P=0$; describing dust or dark matter. The way in which we can obtain dark energy from tachyons is discussed in section III through the incorporation of a reheating phase. During this period, that occurs after inflation, the tachyon energy density decreases many orders of magnitude enabling both have a consistent cosmology [11] and also to get the possibility of a new accelerated expansion phase. The main contribution of our paper is in section IV. There we describe the model and its relation with the two previous sections. In particular we concentrate in the analog model of Quintessential Inflation where the scalar field here is played here by the Tachyon. I end the paper with a summary.

\section{TACHYONS AND INFLATION}

Let us consider the cosmological consequences of a matter component given by the Tachyon through the Born-Infeld action

$$
S=\int \sqrt{-g} d^{4} x\left[\frac{R}{16 \pi G}-V(\phi) \sqrt{1-g^{\mu \nu} \partial_{\mu} \phi \partial_{\nu} \phi}\right]
$$

where $\phi$ is a scalar field associated with the Tachyon. We have to stress here that this action has not been derived from first principles. In fact, the way in which the Tachyon appears coupled to gravity leads also to many other terms that are not in our functional $S$. Actually it can be considered as a good starting point in the study of Tachyons in curved space [6]. We work in a $3+1$ space-time with a flat FRW metric

$$
d s^{2}=d t^{2}-a(t)^{2} d \mathbf{x}^{2}
$$


where $a(t)$ is the scale factor. By computing the stressenergy tensor we find that the Tachyon can be interpreted as a fluid with energy density

$$
\rho=\frac{V(\phi)}{\sqrt{1-\Pi^{2}}}
$$

and a negative density pressure

$$
p=-V(\phi) \sqrt{1-\Pi^{2}}
$$

where $\Pi=\dot{\phi}$ and we have assumed that $\phi$ is a homogeneous field. The equation of state of this component is $p=\left(\Pi^{2}-1\right) \rho$ which for a constant potential value is equivalent to the Chaplygin gas EoS.

The equation of motion for the scalar field is

$$
\dot{\Pi}+\left(3 H \Pi+\frac{V^{\prime}}{V}\right)\left(1-\Pi^{2}\right)=0,
$$

where $H=\dot{a} / a$ is the Hubble parameter and a prime means ${ }^{\prime}=d / d \phi$. In general the Friedman equation can be written as

$$
H^{2}=\frac{8 \pi G}{3}\left[\rho_{\phi}+\rho_{m}\right]
$$

The shape of the tachyonic potential depends on the system under consideration; for example, from bosonic string theory the potential has a maximum at $\phi=0$, where that maximum $V=V_{0}$ is the tension of some unstable bosonic D-brane, and a local minimum with $V=0$ usually at $\phi \rightarrow \infty$. In this paper we consider a well motivated expression

$$
V(\phi)=V_{0} \exp \left(-\phi / \phi_{0}\right)
$$

which has been used in Ref. 7] and the parameter $V_{0}$ is proportional to a D3-brane tension. The fact that this tension and also the 4D Planck mass can be expressed in terms of the string parameters, leads to a consistency problem among CMBR observations, gravity waves detection, and inflationary perturbations [8, 9]. Although some potentials can circumvent this problem, (see also [12]) I choose to work with potential (7) and describe the phenomenology associated with a model consistent with observations.

Let us study Inflation in this context. From (5) and the Friedman equation (6) we can derive the equation

$$
\frac{\ddot{a}}{a}=\frac{8 \pi G V}{3 \sqrt{1-\dot{\phi}^{2}}}\left(1-\frac{3}{2} \Pi^{2}\right),
$$

from which we conclude that accelerated expansion occurs if the condition

$$
\Pi^{2}<\frac{2}{3}
$$

is satisfy. From Eqs. (3) and (4) we find that a cosmological constant equation of state regime is reached if initially the scalar field kinetic energy is very small

$$
\Pi^{2} \ll 1
$$

To keep holding these two conditions as much as possible, we need to ensure a slow roll regime of the field. It means a period where

$$
\dot{\Pi} \ll 3 H \Pi\left(1-\Pi^{2}\right) .
$$

From (10) and (11) we find the slow roll equations of the system

$$
\begin{aligned}
& H^{2} \simeq \frac{8 \pi G}{3} V(\phi), \\
& 3 H \Pi+\frac{V^{\prime}}{V} \simeq 0 .
\end{aligned}
$$

From these two equations we can rewrite the condition (10) in terms of the scalar field potential $V(\phi)$ leading to the well known inequality [7, 9]

$$
\frac{\left(V^{\prime}\right)^{2}}{V^{3}} \ll 24 \pi G,
$$

and doing a similar work with the second condition (11) we find

$$
\frac{V^{\prime \prime}}{V^{\prime} \sqrt{V}} \gg \sqrt{24 \pi G} .
$$

We can also compute the number of e-foldings during Inflation. From the equations we have derived we find

$$
N(\phi)=\int H d t=-\int_{\phi}^{\phi_{e n d}} \frac{H^{2} V}{V^{\prime}} d \phi,
$$

which lead us to the expression

$$
N(\phi)=\frac{8 \pi \phi_{0}^{2}}{M_{p}^{2}} V_{0}\left[e^{-\phi_{i} / \phi_{0}}-e^{-\phi_{e} / \phi_{0}}\right],
$$

where $\phi_{i}$ and $\phi_{e}$ are the values of the tachyon field at the beginning and end of the inflationary phase. The factor in front of the parenthesis defines the dimensionless parameter $X_{0}$ introduced by Fairbairn and Tytgat 7 ]. To solve the horizon and flatness problem we need at least a number of 65 e-folds of inflation. Because after inflation the inequality (14) saturates (which is equivalent to the condition $\epsilon \simeq 1$ ) implies (up to factors) $X_{0} \simeq \exp \left(\phi_{e} / \phi_{0}\right)$, so we are safe to neglect the second term in eq.(17). This implies the restriction

$$
16 \pi \frac{V_{0} \phi_{0}^{2}}{M_{p}^{2}} e^{-\phi_{i} / \phi_{0}}>65 .
$$

Neglecting the exponential, the inequality implies that this factor follows as in Ref. 7]. The inclusion of the exponential, which depends on the value $\phi_{i}$, enable us to both satisfy the restriction (18) and also the one we shall find on $\phi_{0}$ at the end of section IV. 


\section{REHEATING THE UNIVERSE}

In a universe filled only by tachyons, the evolution of the scale factor after inflation ends asymptotically as $a(t) \sim t^{2 / 3}$, implying that there is no room for a second period of accelerated expansion [5, 6, 13]. A simple way to see that, is to consider the scalar field equation (5). From the beginning of inflation $\Pi$ increases with time, $\dot{\Pi}>0$. The condition for that is

$$
3 H \Pi<\frac{1}{\phi_{0}},
$$

where we have used (7) in (5). Because $H$ is a decreasing function of time, $\Pi$ increases slowly and $\phi_{0}$ is a constant, the tachyon field never stop rolling down its potential towards its asymptotic value $\Pi \rightarrow+1$, which leads to $p \sim 0$ from Eq. (4). However, to make contact with the standard model of cosmology, we need a radiation dominated period after inflation, and after that a matter dominated phase appropriated for the large scale structure formation process. This immediately implies that we have to consider a period of reheating after inflation.

Reheating is the period where almost all particles in the universe were created and it begins just at the end of the inflationary era. In the original Quintessential Inflationary model [2] the reheating proceed through gravitational particle production [3]. In this context the inflaton (the tachyon in this work) does not decay, but it continues rolling down the potential during the radiation and matter dominated phases of the universe. The main problem here is the efficiency of the process.

An alternative mechanism of reheating is the introduction of another scalar field in the model called "curvaton" [14]. In this framework, the reheating is produced by the decay of the curvaton field, meanwhile another field drive inflation or the current acceleration in the case of dark energy models [15]. Although the mechanism is more efficient, the all idea is at odds with the quintessential inflationary picture: the use of a single field to describe both regimes.

Another way to reheat the universe is considering the decay of the inflaton itself. In order to model that, we need to coupled the tachyon field with other fields (bosonic and fermionic). Actually, this is the way in which most of the inflationary models makes the transition to a radiation phase [1]. The advantage of this approach, is that the energy density of the tachyon can be reduced through its transformation into particles, enable us to make the model consistent with observations [1], because the tachyon energy density is too large to be compatible with cosmology. In fact, to have a tachyon field $\phi$ relevant today, we must have a density parameter $\Omega_{\phi} \simeq O(1)$. Following 11$]$

$$
\frac{V_{i}}{\phi_{i}^{4}} \sim \frac{\rho_{\text {today }}}{\phi_{\text {today }}^{3} \phi_{i}} \sim \frac{100 \mathrm{Gev} \Omega_{\phi 0}}{\phi_{i}}
$$

where we have assumed that $V_{i}$ is defined at the beginning of the radiation dominated period. Using the expo- nential potential (7), and assuming that $V_{0} \sim M_{p}^{4}$ with $\phi_{i} \sim \mathrm{TeV}$ scale we find that $\phi_{i} / \phi_{0} \sim 23$ implying a fall in 10 orders of magnitude for $\rho_{\phi}$.

In this paper I will assume a simplest phenomenological coupling between the tachyon and radiation, in the spirit of recent works [20, 21, 22]. Let us consider a phenomenological coupling between the tachyon and radiation. By writing the field equation of the tachyon (5) in its fluid form, we get

$$
\dot{\rho_{\phi}}+3 H \Pi^{2} \rho_{\phi}=-\Gamma \Pi^{2} \rho_{\phi},
$$

and the equation for the created relativistic particles

$$
\rho_{m}+4 H \rho_{m}=+\Gamma \Pi^{2} \rho_{\phi}
$$

Here the time scale for particle production $\Gamma^{-1}$ has to be much less than the expansion time scale $H^{-1}$, because we are interested in an efficient reheating process. So we assume that

$$
\Gamma \gg H
$$

During reheating, the equation of motion for the field looks as

$$
\dot{\Pi}+\left(1-\Pi^{2}\right)\left(3 H \Pi+\Gamma \Pi-\frac{1}{\phi_{0}}\right)=0,
$$

which generalized Eq. (5). Because of (23) we assume also that $\Gamma \gg 1 / \phi_{0}$. This assumption is necessary because otherwise, the effects of the particle creation we want to consider would be negligible.

Neglecting the expansion of the universe, the evolution of the tachyon field follows in two stages during reheating; in the first one, the energy density $\rho_{\phi}$ falls down towards a finite value with a $\Pi$ field evolving to the asymptotic value very close to zero. In the second stage, the field $\Pi$ reaches a stable configuration where it keeps the constant value

$$
\Pi_{c} \simeq \frac{1}{\Gamma \phi_{0}} \ll 1
$$

In this case, the tachyon energy density decreases exponentially with time

$$
\rho_{\phi}=V_{0} e^{-\phi / \phi_{0}}
$$

We effectively have an exponential time decay of the energy density, because $\Pi$ is a constant implying that $\phi(t) \propto t$. Once the expression for the energy density $\rho_{\phi}$ has been found, we insert it into the equation for the created particles (22). However, this is not so direct, because the right hand side of (22) is proportional to $\Pi^{2} \rho_{\phi}$. During the first stage of reheating, the field $\Pi$ falls down to $\Pi_{c}$, doing the effect of the particle creation process less efficient. Using this fact and Eq. (3) we notice that we can model the decay of the energy density, during both stages of reheating, as

$$
\rho_{\phi}=M^{4} e^{-\alpha \Delta t}
$$


Using this in (22) the energy density of the created particles behaves approximately

$$
\rho_{m}(t) \simeq \frac{3 \Gamma M^{4} t_{0}}{8}\left[\frac{t}{t_{0}} e^{-\alpha \Delta t}-\left(\frac{t_{0}}{t}\right)^{8 / 3}\right]
$$

where we have assumed that the Hubble parameter behaves as $H \simeq 1 / 2 t$. This assumption is natural because after inflation the tachyon field follows to a matter like dominated universe. Here $t_{0}$ is the time for which $\rho_{m}\left(t_{0}\right)=0$, and marks the beginning of the particle creation process. From this solution we see that the energy density of the created particles reaches a maximum of $\Gamma M^{4} / \alpha$, and then starts to decrease according to a radiation dominated solution. Then during reheating the tachyon equation of state interpolates between $\Pi \sim \sqrt{2 / 3}$, a matter dominated regime and a vacuum one where $\Pi \sim\left(\Gamma \phi_{0}\right)^{-1} \ll 1$. After reheating the universe becomes dominated by radiation, meanwhile the tachyon slowly evolves towards a dust like equation of state (EoS). So the coupling suggested (2122) leads to the correct EoS for a radiation dominated universe. Because $\Pi(t)$ varies during this process, the estimation for the maximum is slightly larger than the exact value.

Using condition (23) in (24) and assuming that $\Delta t \gg$ $\Gamma^{-1}$ we find approximately that

$$
\Pi(t) \sim \Pi_{0} e^{-\Gamma \Delta t}+\frac{1}{\Gamma \phi_{0}}
$$

from which we can write an expression for the tachyon energy density

$$
\rho_{\phi} \simeq \rho_{\phi}\left(t_{0}\right) \exp \left(-\Pi_{0}^{2} \Gamma \Delta t\right)
$$

This result implies that we can use a value $\alpha=\Pi_{0}^{2} \Gamma$ in (27) to obtain the maximum of the radiation energy density $\rho_{m}^{(\max )} \simeq M^{4}$. For this reason, once we solve the problem of doing the tachyon field $\phi$ consistent with observations (leading to a $\Omega_{\phi} \sim O(1)$ ), we also solve the adjustment of the matter component at the end of inflation to obtain a regular standard model of cosmology.

Although the reheating mechanism presented here is inefficient compared to that for a standard scalar field, it can be improved considering the preheating scenario [18. It is a suitable mechanism for a model where the scalar field can not oscillate around the minimum of the potential. During preheating the transfer of energy from the scalar field follows a parametric resonance channel which makes the decay process very fast.

\section{THE TACHYON AS DARK ENERGY}

Using the exponential potential (7), the tachyon field equation of motion (5) admits a transient accelerated expansion solution; a configuration where $\phi \gg \phi_{0}$ and
$\Pi \ll 1$. In terms of the variables defined in [19], similar to those used in Ref.([16]), a configuration where

$$
\lambda \equiv-\frac{M_{p} V^{\prime}(\phi)}{V^{3 / 2}} \leq 1
$$

admits a transient accelerated expansion followed by a deceleration phase for $\lambda \gg 1$. In our case this condition leads to the inequality $\exp \left(\phi / \phi_{0}\right) \leq X_{0}$, which is consistent with the considerations at the end of section II. As we have said in the previous section, during reheating the field $\Pi$ ends with a very small value (25) enabling us to get the appropriated conditions for a second period of exponential expansion. Although the Hubble parameter $H$ decreases during the evolution, after reheating the universe reach the following stage

$$
\Pi<\frac{1}{3 H \phi_{0}}
$$

implying that the field rolls towards $\Pi \rightarrow+1$. A second period of exponential expansion is possible if (see eq. (8) )

$$
\rho_{\phi} \sim \rho_{m}
$$

and the $\Pi$ field must satisfies $\Pi<\sqrt{2 / 3}$. Because at the end of reheating the tachyon energy density is many orders of magnitude less than the matter energy density, the right conditions for a second phase of accelerated expansion can only takes place after the scale factor has growth 10 orders of magnitude; just the right order of magnitude needed to make the tachyon relevant for cosmology. The key observation here is the following; the tachyon energy density evolve always slower than those from radiation and matter. In fact, after reheating the equation of motion for $\phi$ (5) can be written as

$$
\dot{\rho_{\phi}}+3 H \Pi^{2} \rho_{\phi}=0 \text {. }
$$

Because after reheating $\Pi$ is very small, the field evolve slower than matter and radiation. Once the radiation dominated universe has begun, the tachyon equation of motion (5) indicates that, although the slope of $\Pi$ is positive, and the Hubble parameter $H$ decreases with time, the field $\Pi$ takes a long time to get the asymptotic value +1 . It can be see evaluating the time it takes from the initial value $\Pi_{i}$ (the value obtained after reheating) to a final arbitrary configuration (for example $\sqrt{2 / 3}$, the one needed to get a second period of accelerated expansion).

From (5) the slope of $\Pi(t)$ change from a quasi linear regime to an asymptotic one, just at the time when

$$
3 H \Pi \sim \frac{1}{\phi_{0}} .
$$

During the radiation and matter dominated phase, the Hubble parameter $H \sim 1 / t$, so the time scale for the tachyon to reach a value of order one $($ or $\sqrt{2 / 3})$ is

$$
\phi_{0} \sim H^{-1}
$$


that of the Hubble time scale, which is many orders of magnitude larger than the reheating scale $\Gamma^{-1}$ (see eq. (25) and the discussion below eq. (23)). This is the result that indicates the possibility to get a new phase of accelerated expansion today. This time however, would be a transient phase doing the model consistent with the successful standard model of cosmology. The key ingredient is the occurrence of reheating; it not only makes the model consistent with observations 11], but also enable us to have a new period of accelerated expansion.

\section{SUMMARY}

In this paper I have investigated the possibility of using the tachyon as the field driven a quintessential inflationary model. I have described the mechanism to get an inflationary phase and also the process of reheating, necessary to joint it with the standard model of cosmology. The reheating phase becomes crucial in order to both make the tachyon consistent with cosmology and also provide the mechanism to assure a second period of accelerated expansion. I have also found that the recent episode of acceleration is a transient one, after which the universe enters into a matter dominated universe from which they never return. Its important to stress also that the orders of magnitude needed to decrease the tachyon energy density, to make it compatible with the standard cosmology, coincides with the orders of magnitude necessaries to keep the standard Cold Dark Matter model for structure formation intact during the evolution, enabling the tachyon becomes relevant today. The present model alleviates part of the fine tuning in the usual QI model. The adjustment of the scalar field potential slope to get enough inflation and then a long kination phase is not necessary here. Using the tachyon, we have the advantage of using a high energy motivated potential that drives both regimes; inflation and the current acceleration.

\section{Acknowledgments}

VHC want to thanks Ioav Waga and R. Herrera for useful discussions. VHC was supported by DI-UNAB grant 05-04.
[1] A. Linde, Particle Physics and Inflationary Cosmology Harwood, Chur, 1990; Now online at arXiv: hep-th/0503203

[2] P.J.E. Peebles and A. Vilenkin, Phys. Rev. D 59, 063505 (1999); B. Spokoiny, Phys. Lett. B 315, 40 (1993).

[3] L.H. Ford, Phys. Rev. D 35, 2955 (1987).

[4] K. Dimopoulos, Phys. Rev. D 68, 123506 (2003).

[5] A. Sen, Phys. Rev. D 63, 083520 (2001).

[6] G.W. Gibbons, Phys. Lett. B 537, 1 (2002)

[7] M. Fairbairn and M.H.G. Tytgat, Phys. Lett. B 546, 1 (2002).

[8] L. Kofman and A. Linde, JHEP 0207, 004 (2002).

[9] D.A. Steer and F. Vernizzi, Phys. Rev. D 70, 043527 (2004).

[10] S. Nojiri and S. D. Odintsov, Phys. Lett. B 571, 1 (2003); T. Padmanabhan and T. Roy Choudhury, Phys. Rev. D 66, 081301 (2002); see also A. DeBenedictis, A. Das and S. Kloster, Gen. Rel. Grav. 36, 2481 (2004).

[11] G. Shiu, and I. Wasserman, Phys. Lett. B 541, 6 (2002).

[12] Y.S. Piao, R.G. Cai, X. Zhang and Y.Z. Zhang, Phys. Rev. D 66, 121301 (2002).

[13] J.S. Bagla, H.K. Jassal and T. Padmanabhan, Phys. Rev. D 67, 063504 (2003); T. Padmanabhan, Phys. Rev. D 66,
021301 (2002).

[14] D.H. Luth and D. Wands, Phys. Lett. B 524, 5 (2004).

[15] C. Campuzano, S. del Campo, R. Herrera, Phys. Lett. B 633, 149 (2006); ibid Phys. Rev. D 72, 083515 (2005).

[16] JM Aguirregabiria and R Lazkoz, Phys. Rev. D 69, 123502 (2004).

[17] Z.K. Guo, Y.S. Piao, and R.G. Cai, Phys. Rev. D 68, 043508 (2003).

[18] G. Felder, L. Kofman, A. Linde, Phys. Rev. D 59 123523(1999); ibid Phys. Rev. D 60103505 (1999); G. Palma, V.H. Cardenas, Class. Quant. Grav. 18, 2233 (2001); S.E. Joras, V.H. Cardenas, Phys. Rev. D 67 043501 (2003).

[19] E.J. Copeland, M.R. Garousi, M. Sami, and S. Tsujikawa, Phys. Rev. D 71, 043003 (2005).

[20] N. Barnaby and J. M. Cline, Phys. Rev. D 70, 023506 (2004); J.M. Cline, H. Firouzjahi, and P. Martineau, JHEP 0211, 041 (2002).

[21] R. Herrera, D. Pavon, and W. Zimdahl, Gen. Rel. Grav. 36, 2161 (2004).

[22] G. Shiu, S-H.H. Tye, and I. Wasserman, Phys. Rev. D 67, 083517 (2003). 